\title{
Tritium Decay and the Hypothesis of Tachyonic Neutrinos
}

\author{
Jacek Ciborowski \\ Department of Physics, University of Warsaw, ul. Hoża 69, PL-00-681 Warsaw, Poland \\ Jakub Rembieliński \\ Department of Theoretical Physics, University of Eódź, ul. Pomorska 149/153, PL-90-236 Eódź, Poland
}

\begin{abstract}
Numerous recent measurements indicate an excess of counts near the endpoint of the electron energy spectrum in tritium decay. We show that this effect is expected if the neutrino is a tachyon. Results of calculations, based on a unitary (causal) theory of tachyons, are presented. The hypothesis of tachyonic neutrinos also offers a natural explanation of the $\mathrm{V}-\mathrm{A}$ structure of the weak leptonic current in neutrino interactions.
\end{abstract}

\section{INTRODUCTION}

Since several years numerous experiments have been performed with the aim of measuring the electron antineutrino mass in tritium decay, ${ }^{3} \mathrm{H} \rightarrow{ }^{3} \mathrm{He}+e^{-}+\bar{\nu}_{e}$ [1]. This quantity squared, $\xi=m_{\bar{\nu}_{e}}^{2}$, may be determined by fitting the electron energy spectrum near the end point with the formula given below in a simplified form:

$$
\frac{d \Gamma}{d E} \sim p\left(E_{\max }-E\right) \sqrt{\left(E_{\max }-E\right)^{2}-\xi}
$$

where $E\left(E_{\max }\right)$ denote (maximal) energy of the electron in this decay and $p$ its momentum. Surprisingly, all recent experiments yielded negative values of the parameter $\xi$. Owing to the increasing resolution of modern spectrometers, the reason of such results has been attributed to a peculiar unexpected shape of the spectrum in the vicinity of the end point. In qualitative term this phenomenon, hereafter referred to as the end point effect, can be viewed as an excess of counts in that region. This is contrary to common expectations since if the neutrino were massive $(\xi>0)$ a depletion of counts towards the end point would be expected as compared to the massless neutrino case. The enhancement under consideration has been found in the spectra collected at Mainz [1], Troitsk [2] and earlier at LLNL [3]. In particular, numerous measurements performed by the two former groups deliver firm evidence in favour of the effect which may be considered as well established experimentally. Attempts to determine the electron neutrino mass using formula (11), without additional assumptions concerning the origin of the enhancement, lead to doubtful results in these circumstances.

The end point effect has not been convincingly explained on conventional grounds. Both Mainz and Troitsk groups made significant efforts towards understanding their apparatus, data evaluation methods and considered a wide class of related physical phenomena. Moreover, dedicated studies have demonstrated that the end point effect could not originate from mistreatment of molecular effects [5]. A possibility that it might be related to methods of evaluating the data near the end of the physical region has also been considered [6]. Lack of a credible explanation of the effect made room for unconventional hypotheses [7].

In this paper we present calculations of the electron energy spectrum in tritium decay assuming that the neutrino is a tachyon. It must be stressed that changing the sign in front of the parameter $\xi$ in (11) does not at all convert it to the correct formula describing a beta decay spectrum with a tachyonic neutrino.

A tachyon is a particle which moves with velocities always greater than $c$, relative to any reference frame. The energy-momentum relation reads: $E^{2}-\vec{p}^{2}=-\kappa^{2}$, where $\kappa$ will be called the tachyonic mass. Tachyons cannot be described within the framework of the Einstein-Poincaré (EP) relativity because of causality violation (this has been a repeated argument to reject them as possibly existing particles). It proved also impossible to construct a unitary field theory of tachyons on these grounds. The unitary (causal) theory of tachyons proposed recently [8, free of these difficulties, is the basis for calculations presented in this paper. This theory does not invalidate nor modify the EP theory of relativity for massive and light-like particles. In what follows the term 'neutrino' stands for 'electron neutrino' or 'electron antineutrino'. We use the following symbols: total particle energy (momentum $)-E(p)$; kinetic energy - $T$; end point energy $E_{\max }, T_{\max }$, respectively.

\section{THEORY OF SPIN $-\frac{1}{2}$ TACHYONS}

Since the time synchronisation scheme is a convention in the EP relativity, therefore there is a freedom in the definition of the coordinate time. The standard choice is the Einstein-Poincaré synchronisation with the one-way velocity of light isotropic and constant. This choice leads to the well known form of the Lorentz group transformations but the EP coordinate time implies covariant causality for time-like and light-like trajectories only. In order to describe tachyons a different synchronisation scheme must be chosen, namely that of ChangTangherlini (CT), preserving invariance of the notion of the instant-time hyperplane [9]. In this synchronisation scheme the notion of causality is universal, i.e. space-like trajectories (tachyons) are physically admissible too, the 
only inconvenience being that the Lorentz group transformations, which incorporate transformation rules for the velocity of a distinguished (preferred) reference frame, have a more complicated form. The EP and $C T$ descriptions are equivalent for the time-like and light-like trajectories; however a consistent (causal) description of tachyons is possible only in the CT scheme. A very important consequence is that if tachyons exist then the relativity principle is broken, i.e. there exists a preferred frame of reference, however the Lorentz symmetry is preserved. The interrelation between EP $\left(x_{\mathrm{EP}}\right)$ and CT $(x)$ coordinates reads:

$$
x_{\mathrm{EP}}^{0}=x^{0}+u^{0} \vec{u} \vec{x}, \quad \vec{x}_{\mathrm{EP}}=\vec{x},
$$

where $u^{\mu}$ is the four-velocity of the privileged frame as seen from the frame $\left(x^{\mu}\right)$. On the basis of these considerations a fully consistent, Poincaré covariant quantum field theory of tachyons has also been proposed [8]. In the case of the fermionic tachyon with helicity $\frac{1}{2}$ the corresponding free field equation reads:

$$
\left(\gamma^{5}(i \gamma \partial)-\kappa\right) \psi=0
$$

where the bispinor field $\psi$ is simultaneously an eigenvector of the helicity operator with the eigenvalue $\frac{1}{2}$. The elementary tachyonic states are thus labelled by helicity. The $\gamma$-matrices are expressed by the standard ones in analogy to the relations (2). The solution of the equation (3) is given by:

$$
\begin{array}{r}
\psi(x, u)= \\
\begin{array}{r}
\frac{1}{(2 \pi)^{\frac{3}{2}}} \int d^{4} k \delta\left(k^{2}+\kappa^{2}\right) \theta\left(k^{0}\right)\left[w(k, u) e^{i k x} b^{\dagger}(k)\right. \\
\left.+v(k, u) e^{-i k x} a(k)\right],
\end{array}
\end{array}
$$

where the operators $a$ and $b$ correspond to neutrino and antineutrino, respectively. The amplitudes $v$ and $w$ satisfy the following conditions:

$$
\begin{gathered}
w(k, u) \bar{w}(k, u)=\left(\kappa-\gamma^{5} k \gamma\right) \frac{1}{2}\left(1-\frac{\gamma^{5}[k \gamma, u \gamma]}{2 \sqrt{q^{2}+\kappa^{2}}}\right) \\
v(k, u) \bar{v}(k, u)=-\left(\kappa+\gamma^{5} k \gamma\right) \frac{1}{2}\left(1-\frac{\gamma^{5}[k \gamma, u \gamma]}{2 \sqrt{q^{2}+\kappa^{2}}}\right) \\
\bar{w}(k, u) \gamma^{5} u \gamma w(k, u)=\bar{v}(k, u) \gamma^{5} u \gamma v(k, u)=2 q \\
\bar{w}\left(k^{\Pi}, u\right) \gamma^{5} u \gamma v(k, u)=0 .
\end{gathered}
$$

Here $q=u_{\mu} k^{\mu}$ is equal to the energy of the tachyon in the preferred frame and $\Pi$ denotes the space inversion operation. In the massless limit, $\kappa \rightarrow 0$, the above relations are identical with those obtained in the Weyl's theory. An equation similar in its form to (3) has already been proposed in the EP synchronization [10] however the theory based on the latter is not unitary.

\section{BETA DECAY WITH A TACHYONIC ELECTRON NEUTRINO}

\section{A. Amplitude}

On the grounds of the formalism presented in sec. II we calculate the amplitude for a $\beta$ decay, $n \rightarrow p^{+}+$ $e^{-}+\bar{\nu}_{e}$, with a tachyonic electron antineutrino, using an effective four-fermion interaction. In the rest frame of the decaying particle the decay rate for this process reads:

$$
d \Gamma=\frac{1}{4 m_{n}(2 \pi)^{5}} d \Phi_{3}|M|^{2},
$$

where $d \Phi_{3}$ is the phase-space volume element:

$$
\begin{array}{r}
d \Phi_{3}=\theta\left(k^{0}\right) \theta\left(l^{0}\right) \theta\left(r^{0}\right) \delta\left(k^{2}-m_{p}^{2}\right) \delta\left(l^{2}-m_{e}^{2}\right) \times \\
\delta\left(r^{2}+\kappa^{2}\right) \delta^{4}(p-k-l-r) d^{4} k d^{4} l d^{4} r
\end{array}
$$

The amplitude squared, $|M|^{2}$, can be derived (on the tree level) directly from the lepton-hadron part of the effective Fermi weak-interaction Lagrangian:

$$
\mathcal{L}_{\mathcal{I}}=-\left.\mathcal{G}_{\mathcal{F}}\right|_{\mu} \mathcal{J}^{\mu}
$$

where $j_{\mu}$ and $J^{\mu}$ denote leptonic and hadronic currents, respectively. However, under the condition that in the limit of the zero neutrino mass the leptonic current takes the standard $\mathrm{V}-\mathrm{A}$ form, we have two natural choices, which we denote helicity and chirality coupling. Namely, we can choose the corresponding part of the leptonic current in the form:

$$
\bar{u}_{e} \gamma^{\mu} w \quad \text { (helicity coupling) }
$$

or

$$
\bar{u}_{e} \gamma^{\mu} \frac{1}{2}\left(1-\gamma^{5}\right) w \quad \text { (chirality coupling), }
$$

respectively. In the former case the square of the amplitude $\left(M \equiv M_{\mathrm{h}}\right)$ reads:

$$
\begin{aligned}
& \left|M_{\mathrm{h}}\right|^{2}=2 G_{F}^{2} \operatorname{Tr}\left[u_{e} \bar{u}_{e} \gamma^{\mu} w \bar{w} \gamma^{\nu}\right] \times \\
& \quad \operatorname{Tr}\left[u_{p} \bar{u}_{p} \gamma_{\mu}\left(1-g_{A} \gamma^{5}\right) u_{n} \bar{u}_{n} \gamma_{\nu}\left(1-g_{A} \gamma^{5}\right)\right],
\end{aligned}
$$

while in the latter case (chirality coupling: $M \equiv M_{\mathrm{ch}}$ ):

$$
\begin{gathered}
\left|M_{\mathrm{ch}}\right|^{2}=2 G_{F}^{2} \operatorname{Tr}\left[u_{e} \bar{u}_{e} \gamma^{\mu} \frac{1}{2}\left(1-\gamma^{5}\right) w \bar{w} \gamma^{\nu} \frac{1}{2}\left(1-\gamma^{5}\right)\right] \times \\
\operatorname{Tr}\left[u_{p} \bar{u}_{p} \gamma_{\mu}\left(1-g_{A} \gamma^{5}\right) u_{n} \bar{u}_{n} \gamma_{\nu}\left(1-g_{A} \gamma^{5}\right)\right] .
\end{gathered}
$$

Here $p, k, l, r$ are the four-momenta of $n, p^{+}, e^{-}$and $\bar{\nu}$ respectively; the corresponding masses are denoted by $m_{n}, m_{p}, m_{e}$ and $\kappa . G_{F}$ and $g_{A}$ are the Fermi constant and the axial coupling constant. The amplitudes $u_{n}$, 
$\bar{u}_{n}, u_{p}, \bar{u}_{p}, u_{e}, \bar{u}_{e}$ satisfy usual polarisation relations: $u_{n} \bar{u}_{n}=p \gamma+m_{n}, u_{p} \bar{u}_{p}=k \gamma+m_{p}, u_{e} \bar{u}_{e}=l \gamma+m_{e}$, whereas $w \bar{w}$ is given by Eq. (5). After elementary calculations Eqs. (14), (15) read:

$$
\begin{aligned}
\left|M_{\mathrm{h}}\right|^{2}= & 16 G_{F}^{2} \times \\
\times & \left\{[ m _ { n } m _ { p } ( 1 - g _ { A } ^ { 2 } ) - k p ( 1 + g _ { A } ^ { 2 } ) ] \left(4 m_{e} \kappa\right.\right. \\
& -\frac{1}{\sqrt{(u r)^{2}+\kappa^{2}}}\left[4\left(\kappa^{2} l u+l r \cdot u r\right)\right. \\
& \left.\left.-2 \kappa^{2} l u-2 u r \cdot l r\right]\right) \\
+ & \left(1+g_{A}^{2}\right)\left(2 m_{e} \kappa(p k)\right. \\
& -\frac{1}{\sqrt{(u r)^{2}+\kappa^{2}}}\left[2 p k\left(k^{2} l u+l r \cdot u r\right)\right. \\
& \left.\left.-2 \kappa^{2}(p l \cdot u k+k l \cdot u p)-2 u r(p l \cdot k r+k l \cdot p r)\right]\right) \\
+ & 4 g_{A}(p r \cdot k l-p l \cdot k r \\
+ & \left.\left.\frac{m_{e} \kappa}{\sqrt{(u r)^{2}+\kappa^{2}}}(k r \cdot u p-p r \cdot u k)\right)\right\}
\end{aligned}
$$

and

$$
\begin{aligned}
\left|M_{\mathrm{ch}}\right|^{2}= & 16 G_{F}^{2} \times \\
\times & \left\{( 1 + \frac { u r } { \sqrt { ( u r ) ^ { 2 } + \kappa ^ { 2 } } } ) \left[\left(g_{A}^{2}-1\right) m_{n} m_{p} l r\right.\right. \\
& +\left(g_{A}^{2}+1\right)(l p \cdot k r+p r \cdot k l) \\
& \left.+2 g_{A}(k l \cdot p r-l p \cdot k r)\right] \\
+ & \frac{\kappa^{2}}{\sqrt{(u r)^{2}+\kappa^{2}}}\left[\left(g_{A}^{2}-1\right) m_{n} m_{p} u l\right. \\
& +\left(g_{A}^{2}+1\right)(l p \cdot u k+u p \cdot k l) \\
& \left.\left.+2 g_{A}(k l \cdot u p-l p \cdot u k)\right]\right\}
\end{aligned}
$$

In order to calculate the differential energy spectrum of electrons in the $\beta$ decay with a tachyonic electron neutrino, $d \Gamma / d l^{0}$, it is necessary to account for the velocity of the preferred frame, $u$. We take $u_{\mu}=(1,0,0,0)$ for simplicity, i.e. we derive the result in a reference frame which is at rest with respect to the preferred frame (consequences of a non-negligible velocity of the preferred frame are discussed in Sec. [V]). The spectrum $d \Gamma / d l^{0}$ may be obtained for both considered cases (helicity and chirality coupling) by means of formulae (9), (10), (16) and (17), after elementary integration with respect to

${ }^{*}$ But with $\gamma$ in the CT synchronisation 8]. $d^{4} k d^{3} \vec{l} d^{3} \vec{r}$, from the following formula (which gives identical results in the limit $\kappa^{2} \rightarrow 0$ as that for the massless neutrino):

$$
\frac{d \Gamma}{d l^{0}}=\frac{1}{128 \pi^{3} m_{n}} \int_{\max \left(r_{-}, 0\right)}^{r_{+}} d r^{0}\left|M\left(l^{0}, r^{0}\right)\right|^{2}
$$

with

$$
\begin{gathered}
r_{ \pm}=\left\{-\Delta m^{2} l^{0}+\Delta m^{2} m_{n}+2\left(l^{0}\right)^{2} m_{n}-2 l^{0} m_{n}^{2}\right. \\
\pm \sqrt{\left(l^{0}\right)^{2}-m_{e}^{2}}\left[\left(\Delta m^{2}\right)^{2}+4 \kappa^{2} m_{e}^{2}-4 \Delta m^{2} l^{0} m_{n}\right. \\
\left.\left.-8 \kappa^{2} l^{0} m_{n}+4 \kappa^{2} m_{n}^{2}+4\left(l^{0}\right)^{2} m_{n}^{2}\right]^{\frac{1}{2}}\right\} \times \\
\times\left[2\left(m_{e}^{2}-2 l^{0} m_{n}+m_{n}^{2}\right)\right]^{-1} .
\end{gathered}
$$

Here $|M|^{2}=\left|M_{\mathrm{h}}\right|^{2}$ or $\left|M_{\mathrm{ch}}\right|^{2}$, respectively, and $u l=l^{0}$, $u r=r^{0}, u p=m_{n}, u k=m_{n}-l^{0}-r^{0}, k p=m_{n}\left(m_{n}-l^{0}-\right.$ $\left.r^{0}\right), k l=-m_{n} r^{0}-m_{e}^{2}+\frac{1}{2} \Delta m^{2}, k r=-m_{n} l^{0}+\kappa^{2}+\frac{1}{2} \Delta m^{2}$, $p r=m_{n} r^{0}, l p=m_{n} l^{0}, l r=m_{n}\left(l^{0}+r^{0}\right)-\frac{1}{2} \Delta m^{2}$ and $\Delta m^{2}=m_{n}^{2}-m_{p}^{2}+m_{e}^{2}-\kappa^{2}$.

\section{B. Electron energy spectrum}

We have calculated differential electron energy spectra, $d \Gamma / d l^{0}$, in tritium decay using the following values for the masses of ${ }^{3} \mathrm{H}$ and ${ }^{3} \mathrm{He}: m_{n}=2809.94 \mathrm{MeV}$ and $m_{p}=2809.41 \mathrm{MeV}$, respectively (hereafter we use $\left.l^{0} \equiv E\right)$. The corresponding value for the electron end point kinetic energy in the preferred frame is $T_{\max }=18587.56 \mathrm{eV}$. Differential electron energy spectra, $d \Gamma / d E$, corresponding to decays with a massive, massless and tachyonic neutrino, in the vicinity of the endpoint, are shown in Fig. 11a. The tachyonic spectra for both couplings near the end point rise above that for the massless neutrino. Moreover, they terminate at $T=T_{\max }$ with a quasi step: the function $d \Gamma / d E$ decreases linearly to zero over the energy interval of $2 \kappa p_{\max } / m_{n}$ (where $p_{\max }$ denotes the maximal electron momentum) which in the tritium decay amounts to $\approx 10^{-3} \mathrm{eV}$ for $\kappa=8 \mathrm{eV}$. The magnitude (height) of the step depends on the choice of coupling as well as on the value of $\kappa$, as can be seen in Fig. Ila,b.

Thus if the neutrino were a tachyon, one would expect an excess of the counting rate near the end point, i.e. an effect qualitatively similar to the one actually observed. Since the detectors used in Mainz and Troitsk are integrating spectrometers, we integrated the electron energy spectrum given by (18), folding in a simplified experimental resolution function used in these experiments [11], with energy resolution $\Delta E=4 \mathrm{eV}$ (not accounting for the final state energy spectrum). The resulting linearised (cube root) electron energy spectrum near the endpoint is shown in Fig. 2. There is a striking similarity of the predicted shape and that observed in the Troitsk data [2]d (which is the only published linearised spectrum). We 

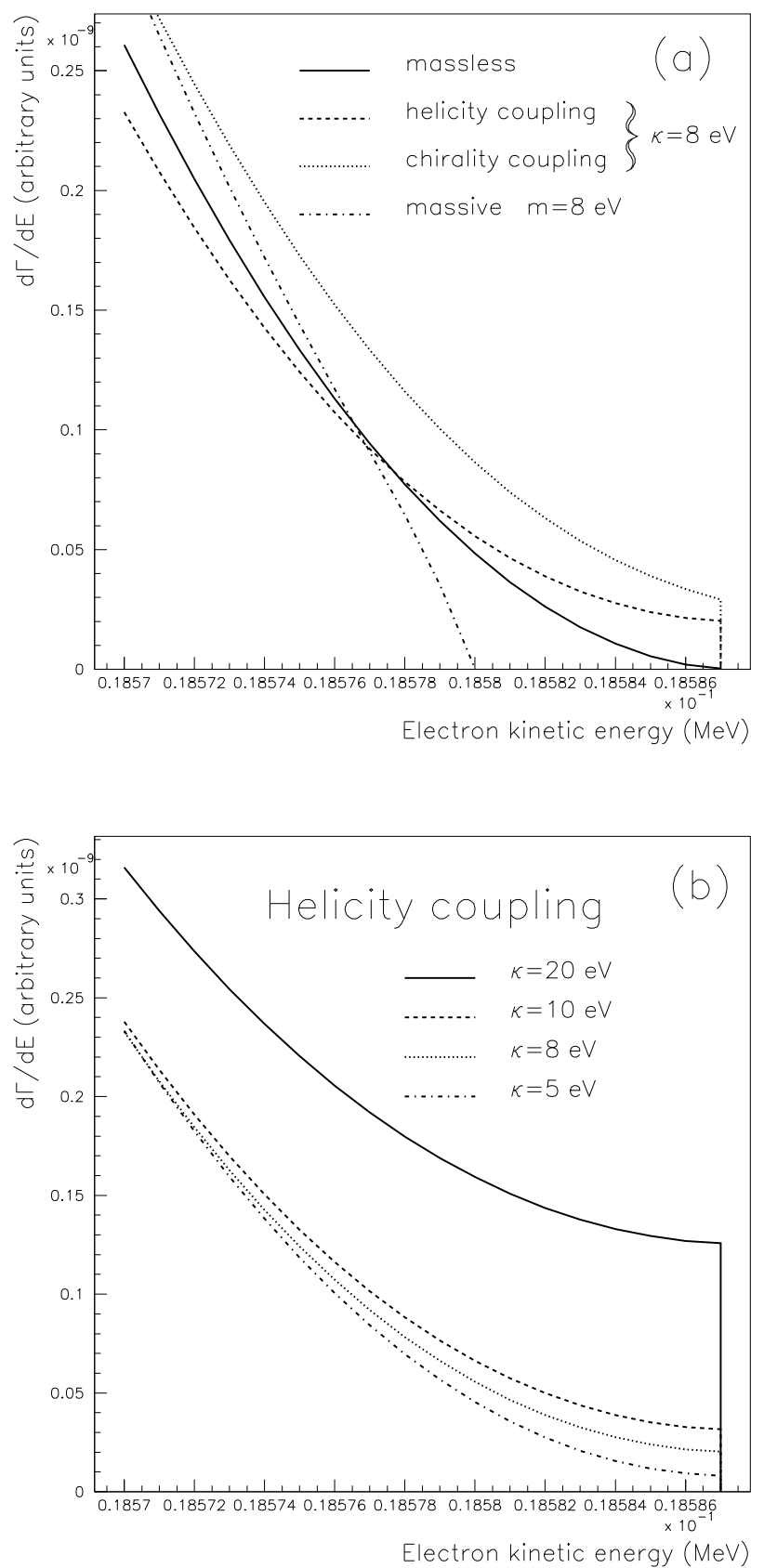

FIG. 1. (a) Differential electron energy spectra in the vicinity of the end point, for tritium decay with: a tachyonic antineutrino of mass $\kappa=8 \mathrm{eV}$, massless neutrino and massive neutrino of mass $m=8 \mathrm{eV}$; (b) as above for a tachyonic electron antineutrino with the helicity coupling, for a range of tachyonic masses, $\kappa$.

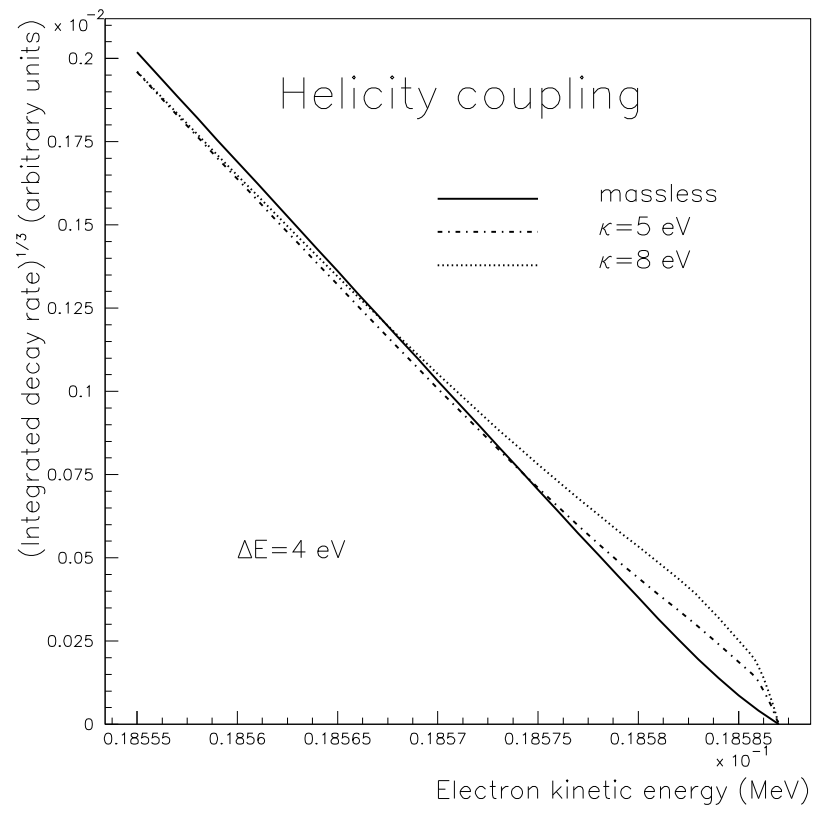

FIG. 2. Linearised (cube root) integral electron energy spectrum with folded experimental resolution function (see text).

also verified that the end point effect of the observed magnitude could have hardly been discovered in earlier measurements which had much poorer energy resolution.

For practical purposes the rigorous but complicated expressions for the electron energy spectra (18) may be approximated in order to write them in terms of the variable $\left(E_{\max }-E\right)$ and the electron momentum $p$. The simplified form, valid under the condition $E \leq E_{\max }$, for the helicity coupling reads:

$$
\begin{aligned}
& \frac{d \Gamma}{d E}= \frac{G_{F}}{2 \pi^{3}}\left[\kappa m_{e}\left(1-3 g_{A}^{2}\right)\right. \\
&\left.+\left(1+3 g_{A}^{2}\right) E \sqrt{\left(E_{\max }-E\right)^{2}+\kappa^{2}}\right] \times \\
& \quad \times p \sqrt{\left(E_{\max }-E\right)^{2}+\kappa^{2}}
\end{aligned}
$$

and for the chirality coupling:

$$
\begin{aligned}
\frac{d \Gamma}{d E}= & \frac{G_{F}}{4 \pi^{3}}\left(1+3 g_{A}^{2}\right) E p\left[\left(E_{\max }-E\right)^{2}\right. \\
& \left.+\left(E_{\max }-E\right) \sqrt{\left(E_{\max }-E\right)^{2}+\kappa^{2}}+\kappa^{2}\right],
\end{aligned}
$$

with the additional explicite condition that $\frac{d \Gamma}{d E}=0$ for $E>E_{\max }$ since the approximated spectra do not vanish at $E=E_{\max }$ (step). 


\section{PREFERRED FRAME AND TIME DEPENDENT EFFECTS}

An interesting property of amplitudes for processes involving tachyonic neutrinos, in particular for the beta decay, is their dependence on the velocity four-vector of the preferred frame, $u$ (Eq. (16)). On the grounds of cosmological considerations one might expect that a frame in which the cosmic microwave background radiation (CMBR) is isotropic is a natural candidate for the preferred frame. In such a case results derived in previous sections are sufficiently precise because the Solar System is almost at rest relatively to the CMBRI.

Consider a certain configuration of the final state particles momenta in a beta decay which occurs in a reference frame moving with velocity $\vec{u}$ with respect to the preferred frame. The maximal kinetic energy of the electron, $T_{\max }$, depends on $\beta=|\vec{u}| / c$ and $\cos \omega$, where $\omega$ is the angle between the neutrino momentum and the vector $\vec{u}$ :

$$
T_{\max }(\beta, \cos \omega)=T_{\max }-\Delta T_{\max }(\beta, \cos \omega)
$$

where

$$
\Delta T_{\max }(\beta, \cos \omega)=\frac{\kappa \beta \cos \omega}{\sqrt{1-\beta^{2} \cos ^{2} \omega}} .
$$

Momenta of the final state particles in the $\beta$ decay are aligned at the end point and thus the angle $\omega$ may be expressed by the angle corresponding to the electron. Assume for simplicity that the electron spectrum is measured in an ideal spectrometer in which electrons are moving along the spectrometer axis. Thus the angle $\omega$ between this axis and the vector $\vec{u}$ changes with time due to Earth's rotation and day - night variations of $T_{\max }$ are expected. If we identify the preferred frame with the $\operatorname{CMBR}\left(\beta \approx 10^{-3}\right)$ we obtain $\Delta T_{\max }<10^{-2} \mathrm{eV}$ for the tachyonic electron neutrino mass of a few $\mathrm{eV}$, i.e. an effect undetectable at present. If however the velocity of the preferred frame were large $(\beta>0.1)$, the expected variation of the end point energy would be of order $\mathrm{eV}$.

\section{SUMMARY AND CONCLUSIONS}

We have shown that the electron energy spectrum in a beta decay with a tachyonic neutrino rises above that for the massless neutrino and ends with a quasi step at $E=E_{\max }$. This feature may explain the excess of counts observed in tritium decay in the vicinity of the end point if the neutrino were a tachyon. Our prediction and the measurement of Troitsk show a remarkable similarity

\footnotetext{
${ }^{\dagger}$ Velocity deduced from the dipole anisotropy in temperature amounts to about $350 \mathrm{~km} / \mathrm{s}[12$.
}

when presented on a linearised plot. Since the neutrino field is an eigenvector of the helicity operator with the eigenvalue $\frac{1}{2}-$ helicity coupling offers a natural explanation of the $V-A$ structure of the weak leptonic current in neutrino interactions. Certain preliminary considerations concerning the hypothesis of tachyonic neutrinos may be found elsewhere 13 .

\section{ACKNOWLEDGEMENT}

We wish to thank K.A. Smoliński and P. Caban for their contribution to numerical calculations; B. Jeziorski for discussions concerning molecular final states; E. Otten, J. Bonn and Ch. Weinheimer for numerous discussions, information about the detector and the results of the Mainz experiment as well as comments concerning the manuscript; V.M. Lobashev and N.A. Titov for information concerning the Troitsk experiment.

[1] Mainz ${ }^{3} \mathrm{~T}$ decay experiment:

a. Ch. Weinheimer, in Proc. XVIII Conf. on Neutrino Physics and Astrophysics (NEUTRINO98), Takayama, Japan, 1998, (to appear)

b. J. Bonn, in Proc. Int. Conf. on High Energy Physics (HEP97), Jerusalem, 1997

c. H. Barth et al., in Proc. of the XVI Workshop on Weak Interactions and Neutrinos (WIN97), Capri, Italy, 1997, Nucl. Phys. B (Proc. Suppl.) 66, 183 (1998)

d. J. Bonn, in Proc. XVII Conf. on Neutrino Physics and Astrophysics (NEUTRINO96), Helsinki, Finland, 1996, p. 259

e. Ch. Weinheimer et al., Phys. Lett. B 300, 210 (1993)

[2] Troitsk ${ }^{3} \mathrm{~T}$ experiment:

a. V.M. Lobashev, in Proc. XVIII Conf. on Neutrino Physics and Astrophysics (NEUTRINO98), Takayama, Japan, 1998, (to appear)

b. V.M. Lobashev, in Proc. of the XVI Workshop on Weak Interactions and Neutrinos (WIN97), Capri, Italy, 1997, Nucl. Phys. B (Proc. Suppl.) 66, 187 (1998)

c. V.M. Lobashev, in Proc. XVII Conf. on Neutrino Physics and Astrophysics (NEUTRINO96), Helsinki, Finland, 1996, p. 264

d. A.I. Belesev et al., Phys. Lett. B 350, 263 (1995)

[3] W. Stoeffl, D. Decman et al., Phys. Rev. Lett. 75, 3237 (1995)

[4] H. C. Sun et al., Chin. J. Nucl. Phys. 15, 261 (1993)

E. Holzschuh et al., Phys. Lett. B 287, 381 (1992)

H. Kawakami et al., Phys. Lett. B 256, 105 (1991)

R.G.H. Robertson et al., Phys. Rev. Lett. 67, 957 (1993)

[5] P. Froelich et al., Phys. Rev. Lett. 71, 2871 (1993) H.C. Sun et al., Int. J. Mod. Phys. A 10, 2841 (1995) Workshop: 'The Tritium $\beta$ Decay Spectrum: The negative $m_{\nu_{e}}^{2}$ Issue', Harvard-Smithsonian Center for Astro- 
physics, Cambridge, Massachusetts, USA, 1996

S. Jonsell, H.J. Monkhorst, Phys. Rev. Lett. 76, 4476

(1996)

B. Jeziorski (private communications)

[6] L.A. Khalfin, PDMI-8/1996 (unpublished)

M. Roos, L.A. Khalfin, HU-TFT-96-13 hep-ex/9605008 (unpublished)

[7] R.N. Mohapatra, S. Nussinov, Phys. Lett. B 398, 63 (1997)

J.I. Collar, hep-ph/9611420 (unpublished)

[8] J. Rembieliński, Phys. Lett. A 78, 33 (1980)

J. Rembieliński, KFT UŁ 2/94 hep-th/9410079 (unpublished)

J. Rembieliński, KFT UŁ 5/94 hep-th/9411230 (unpublished)

J. Rembieliński, Int. J. Mod. Phys. A 12, 1677 (1997)

[9] T. Chang, Phys. Lett. A 70, 1 (1979)

T. Chang, J. Phys. A 12, L203 (1979)

T. Chang, J. Phys. A 13, L207 (1980)

F.R. Tangherlini, N. Cim. Suppl. 20, 1 (1961)

[10] A. Chodos, A.I. Hauser, V.A. Kostelecky, Phys. Lett. B 150, 431 (1985)

[11] Ch. Weinheimer, N.A. Titov (private communications)

[12] P.M. Lubin et al., Phys. Rev. Lett. 50, 616 (1983)

D.J. Fixsen et al., Phys. Rev. Lett. 50, 620 (1983)

[13] J. Ciborowski, J. Rembieliński, in Proc. XXVIII Int. Conf. on High Energy Physics (ICHEP96), Warsaw, 1996, edited by Z. Ajduk, A.K. Wróblewski (World Scientific, Singapore 1997), p. 1247

J. Ciborowski, J. Rembieliński, hep-ph/9607477 (unpublished)

J. Ciborowski, J. Rembieliński, in Proc. Int. Conf. on High Energy Physics (HEP97), Jerusalem, 1997 (to appear) 